\title{
Training and learning for crisis management using a virtual simulation/gaming environment
}

\author{
Warren E. Walker · Jordan Giddings • \\ Stuart Armstrong
}

Received: 9 September 2009/Accepted: 15 January 2011/Published online: 6 March 2011

(C) The Author(s) 2011. This article is published with open access at Springerlink.com

\begin{abstract}
Recent advances in computers, networking, and telecommunications offer new opportunities for using simulation and gaming as methodological tools for improving crisis management. It has become easy to develop virtual environments to support games, to have players at distributed workstations interacting with each other, to have automated controllers supply exogenous events to the players, to enable players to query online data files during the game, and to prepare presentation graphics for use during the game and for post-game debriefings. Videos can be used to present scenario updates to players in "newscast" format and to present pre-taped briefings by experts to players. Organizations responsible for crisis management are already using such technologies in constructing crisis management systems (CMSs) to coordinate response to a crisis, provide decision support during a crisis, and support activities prior to the crisis and after the crisis. If designed with gaming in mind, those same CMSs could be easily used in a simulation mode to play a crisis management game. Such a use of the system would also provide personnel with opportunities to rehearse for real crises using the same tools they would have available to them in a real crisis. In this paper, we provide some background for the use of simulation and gaming in crisis
\end{abstract}

\footnotetext{
W. E. Walker $(\square)$

Delft University of Technology, Delft, The Netherlands

e-mail: w.e.walker@tudelft.nl

J. Giddings

UK Department for Transport, London, UK

e-mail: Jordan.giddings@dft.gsi.gov.uk

S. Armstrong

QinetiQ, Farnborough, UK

e-mail: saarmstrong@qinetiq.com
}

management training, describe an architecture for simulation and gaming, and present a case study to illustrate how virtual environments can be used for crisis management training.

Keywords Simulation - Gaming - Crisis management systems · Virtual environments

\section{Introduction}

Crises are rare events. Each crisis is different from other crises. Among the variables that define a crisis are its type (e.g., flood, explosion, war, airplane crash), location, affected population, and relevant support organizations. These variables are practically impossible to predict in advance. Also, the crisis and the resources available to deal with it are continuously changing over time. The problem that organizations responsible for management of major disaster response activities face is how to deal with these "state-of-the-world" uncertainties in training and preparing to handle crises. Quade (1989) defines state-of-the-world uncertainties as those that are beyond the practical ability of analysts to predict and cannot be reduced to risks. One way to deal with these uncertainties, which we describe in this paper, is to train crisis management personnel in a realistic environment using scenarios and gaming. Similar approaches have been suggested in the past. Kleiboer (1997) reviews the literature on crisis simulations and concludes that crisis simulations "help plan for crisis management... [They] offer a close approximation of the stress and flow of events of a real-world crisis". And Walker et al. (1989) say "Computer-based simulators can bridge the gap between classroom training and live simulations and provide the management training needed". 
Recent advances in computers, networking, and telecommunications offer new opportunities for using simulation and gaming as methodological tools for improving crisis management. As suggested by Mendonça et al. (2006), the degree of realism in a game is important for enhancing the educational benefits of a simulation. Beroggi et al. (1995) explain that virtual environments can provide the sensation of a real emergency situation, which is difficult to create in traditional simulation and gaming situations. Tate et al. (1997) describe the US Navy's Virtual Environment Training Technology (VETT) program for shipboard firefighter mission preparation, rehearsal, and training. They conclude that virtual environments provide "a flexible, synthetic environment where firefighters can familiarize themselves with an unfamiliar part of the ship, practice firefighting procedures by interacting with simulated fire and smoke, and test firefighting tactics and strategies without risking lives or property".

In the remainder of the paper, we describe the use of simulation and gaming in crisis management and show how new developments in information technology and communications can be used to create more realistic situations and lead to new tools for educating crisis managers to respond to and manage crises.

\section{Features of a crisis}

A crisis can be considered to be a transition in a system or related set of systems that is triggered by unpredictable events requiring action and response to enable stability within the system to be re-established. While each crisis will have unique variables and outcomes, all can be considered to act through a similar time cycle and evolution, as indicated in Fig. 1. The response to a crisis goes through a cycle of initial response, a period of consolidation, through a period of restoration to a stable system. It should be noted that the state of the resulting stable system may be quite different from the state prior to the crisis (e.g., the US post September 11, 2001, New Orleans post Hurricane Katrina).

Throughout the evolution of a crisis, decisions need to be taken, dependent upon the nature of the crisis. This will typically require information to be extracted from the situation before the crisis, collected during the crisis to support decisions, and retained after the crisis to enable experiences to be learned from in order to enhance resilience against future events. Equally, well-controlled and informed decision making throughout the evolution of a crisis can minimize the cost and level of response required, reduce the time taken for restoration to normality, and potentially even trigger a response before the initial event has taken place (for example, weather forecasting, monitoring for pandemic influenza, unusual behavior patterns indicative of terrorist activity). The positive impact of such decision making is indicated by the blue line in Fig. 1. The dotted line indicates the need to collect information during the evolution of the crisis for future reference, forensics, and learning from experience to enhance the resilience of the system to future events.

\section{Scenarios}

Scenarios can play an important role in crisis management and contingency planning. The use of the term "scenario" as an analytical tool dates from the early 1960s, when researchers at the RAND Corporation defined states of the world within which alternative weapons systems or military strategies would have to perform. Since then, their use has grown rapidly, and the meanings and uses of scenarios

Fig. 1 Evolution of a crisis

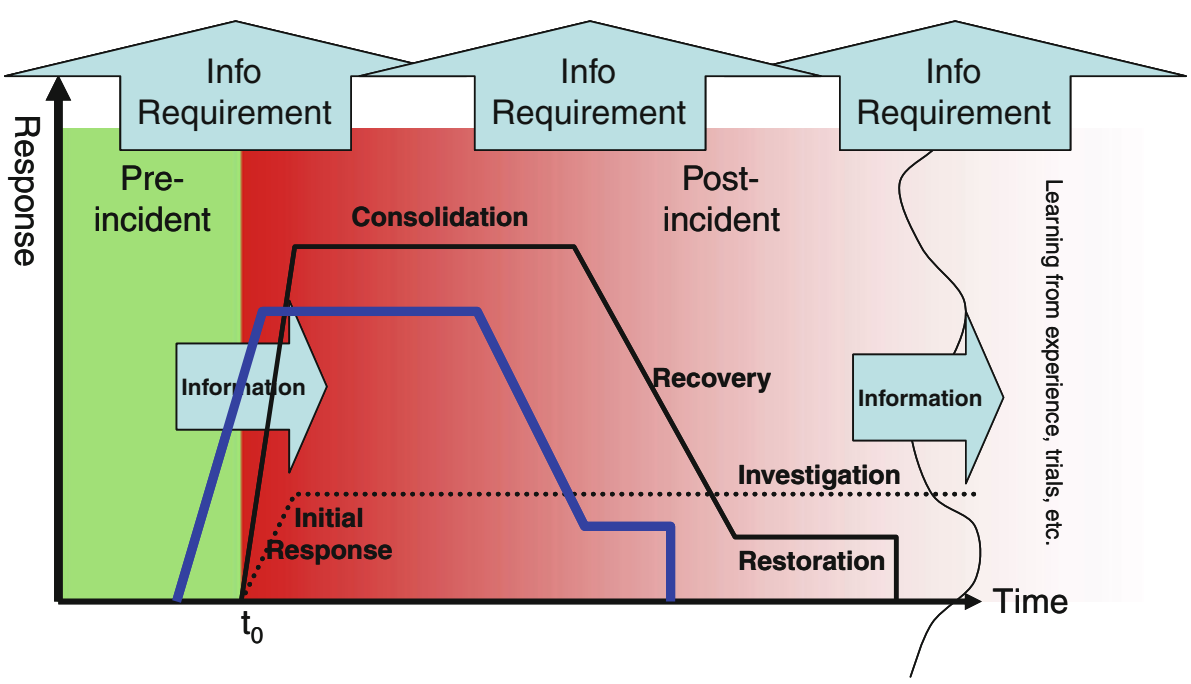


have become increasingly varied. As a consequence, misunderstandings and communication problems about scenarios can arise easily. We, therefore, wish to begin by defining what we mean by a scenario in the context of crisis management. For the purposes of this paper, we propose the following definition of a crisis management scenario, which is based on a more general definition suggested by Quade (1989).

A description of the conditions under which the crisis management system or crisis management policy to be designed, tested, or evaluated is assumed to perform.

The terms "context", "setting", "situation", or "environment" are often used loosely as equivalents to the term "scenario", but we make some distinctions among them. In particular, we propose to split a crisis management scenario into two parts- the context and the crisis. The context may be described as the overall background or environment within which the specific crisis is to be considered (the area shown in green in Fig. 1). It is the state of the affected area at the time of the crisis. For example, it would define, for a given point in time, the demographics, the geography, the organizational relationships, the availability of data, the telecommunications situation, etc. The context is an environmental framework into which many different crises might be embedded for study,

The crisis, on the other hand, is a script for the specific crisis. It includes the chain of (potentially hypothetical) events that lead up to the crisis. If gaming is to be used to examine possible responses (as we propose here), it would also include the exogenous events during the period of the game (the chain of events outside the control of players in the system). For example, the crisis would describe the weather, political events, etc., before, during, and after the specific crisis. It is the sequence of events to which the crisis management system must respond. For a flood, it would specify the times and places where specific dikes were breached, services were disrupted, persons were swept away, etc.

The context plus the crisis prior to the start of the game would provide the players with the necessary background information about the situation to enable them to specify the initial conditions for their response activities. Also, of course, the purpose of the game would provide several parameters that are vital to the scenario, such as the setting, potential list of actors, and many of the "rules of the game". What we propose in this paper is the use of realtime information (either captured from historical events or in managing a crisis in real time) to provide both the context and the crisis script to enable significantly enhanced training and crisis management capability.
3.1 Why use scenarios?

A scenario describes a hypothetical, but plausible, situation that may be used for a variety of purposes. DeWeerd (1967) lists four purposes of scenarios that are relevant for crisis management:

- To supply the starting point and exogenous events for a game.

- To supply a consistent and plausible situation as a background for the discussion of a proposed crisis management system or crisis management policies.

- As an environment in which to examine the functioning of various [response] strategies.

- As a background for contingency planning.

Scenarios are primarily communication devices. Although they do not reduce the uncertainties inherent in describing a future state of the world, they make situations more concrete, so users can treat a proposed response strategy or crisis management system within a self-consistent and plausible set of circumstances. Deweerd quotes Herman Kahn as saying scenarios are meant to "stretch the mind and force a planner to envisage the future in concrete terms". And Quade (1985) points out that well-formulated scenarios have proved useful in broadening the number of contingencies taken seriously in military and industrial planning "by forcibly illustrating the advantages or pitfalls of various proposals or of a new capability".

A scenario specifies a possible, but not necessarily probable, context and series of events. Its usefulness for crisis management planning or decision making does not depend on its accuracy or the probability of its occurrence. This is somewhat different from its usefulness for policy analysis purposes, where the probability of the situation occurring can be an important factor. In fact, when using scenarios, it is crucial to keep in mind that they are not predictions. The treatment of a scenario as a prediction is one of the most common mistakes made by both their creators and their users, and time should not be spent arguing about the relative probabilities of occurrence of alternative scenarios. The scenario creator should not be claiming that the events he is describing are likely, but that they are not completely impossible, and that they are plausible enough to be taken seriously by contingency planners. We consider that a range of scenarios developed and driven by real-time events can increase the fidelity and realism of games, but will always remain as excursions from the "real world" to enable new capabilities to be tested, new ways of operating to be tested, and crucially as potentially real-time decision support tools enabling a range of possible time lines to be tested for the most appropriate ways to deal with a developing crisis. 
There is no general theory that allows us to assess scenario adequacy or quality. There are, however, a number of criteria that are often mentioned in the literature as being important. Schwarz (1988) gives a brief summary of them. The most important of these in the crisis management context are consistency, plausibility, credibility, and relevance. Consistency simply means that the assumptions made are not self-contradictory. It is not easy to create a future that is internally and externally consistent (novelists and filmmakers also find it difficult). But inconsistency in a scenario will raise questions about its validity and usefulness. One of the early tests of the validity of a scenario was whether a sequence of events could be constructed that would lead from the present state of the world to the scenario's future state. According to Helmer (1966), although the purpose of a scenario is not to predict the future, "it nevertheless sets out to demonstrate the possibility of a certain future state of affairs by exhibiting a reasonable chain of events that might lead to it". As DeWeerd (1967) states, "In a good scenario there should be no great unexplained leaps, no uninvented weapons, no reversal of the laws of gravity, and no inner contradictions".

The term "plausible" is used to distinguish a scenario from a prediction. It is a statement that the posited chain of events might happen, not will happen. Although the specific events may be highly unlikely to occur at the times indicated or the levels assumed, if a scenario is plausible, then a similar sequence of events might very well occur. Plausibility is a great virtue in a scenario, but, as Kahn and Wiener (1967) point out, the scenario writer should not limit himself to the most plausible possibilities, since "history is likely to write scenarios that most observers would find implausible not only prospectively but sometimes, even, in retrospect”. For example, DeWeerd (1973) notes that "a scenario of the Watergate bugging attempt, written before 1972, would have been held up to scorn by "reasonable" men, as would a scenario written before the Pearl Harbour attack, the Berlin Blockade, the Berlin Wall, or the Cuban Missile crisis". We could equally say the same with the events triggered by the September 11 attacks in the US, Hurricane Katrina, or the global credit crunch.

Credibility is closely related to plausibility. For a scenario to be credible, each change from the present circumstances or those existing at a previous step in the chain should be explained. It is all right to predict that some unforeseen events will occur. However, in mentioning a particular event, it is important to understand why it occurs. If it does occur by "accident", its role in the scenario may not be too important. Otherwise, the scenario loses its credibility and some potential users might refuse to use it. In some cases, the purpose of the scenario should take precedence over credibility (see the discussion of "relevance" below). But such departures from reality should be explicitly noted.

To be useful, the form, role, and content of a scenario must have relevance to the problem at hand. For contingency planning for crises, for example, it might be worthwhile to posit what is usually regarded as irrational behavior by a perpetrator or a development that may be extremely unlikely but would have important, possibly dangerous, consequences.

\subsection{The design of scenarios}

Since scenarios may have many uses, the quality and usefulness of a scenario can only be judged according to the use to which the scenario will be put. That is, the form and content of a scenario has to be determined by the specific task at hand. If being designed as input to a game, the purpose and structure of the game will dictate many of the elements of the scenario. Also, the boundaries of the games (what is endogenous and what is exogenous) will dictate the boundaries for the scenario. This information will provide the setting, the geography and demography, the list of players, the "rules of the game", and other parameters that are vital to the scenario.

Although it is impossible to generalize about how to set the boundaries of a scenario or what form they should take, deLeon (1975) suggests four decisions that are important in the design of any gaming scenario:

- Time setting. For crisis management games, the time should be the present. What we want to determine is how the current crisis response system is working and what can be done to improve it.

- Environmental setting. The environment should be as little changed from the current world as possible. Aspects to be covered include demographic distributions, geographic descriptions, and as much additional information that the players should know in order to make their decisions.

- Level of detail. The conditions for the geographical area where the crisis occurs should be carefully delineated and described. However, the amount of detail should be limited to only what is necessary. Players can only absorb and manipulate a limited amount of information; to overload them with trivia would be self-defeating for the purposes of the game.

- Knowledge, experience, and sophistication of players. The fewer skills, background, and knowledge the players bring to the game, the more thorough the scenario must be. In the games we are proposing, the scenarios will be increasingly driven from realworld contexts, thus enhancing the reality and fidelity 
of the gaming environment and enabling significant levels of verification and validation.

\section{Gaming and simulation}

Abt (1970) defines a game as "an activity among two or more independent decision makers seeking to achieve their objectives in some limiting context". War games date at least as far back as 500 B.C., when the oriental general Sun Tzu is reported to have said "the general who wins a battle makes many calculations in his temple ere the battle is fought" (Weiner 1964, p. 217). Man-machine simulations and war games (utilizing computers to play one of the sides) began to be used in the early 1960s at the RAND Corporation to study real-world political-military crises. For discussions of the techniques they used and situations they considered, see Geisler and Ginsberg (1965) and Shubik and Brewer (1972). Since then, the use of games for a variety of teaching, training, and research purposes has mushroomed. They have helped in developing military strategies, in pre-testing government policies before implementation, and in helping to understand operational complexities in many contexts. Schwabe (1994) provides a brief introduction to gaming as an analytical tool. Much of the information in this section is drawn from his paper.

Traditionally, most games have two or more players, each representing a decision-making entity. For the purpose of this paper, a "player" will be considered as an "intelligent agent" that may be represented by a real individual within the game or by a (semi)autonomous agent driven by a computer or real-world feed. Each player is typically assigned a specific role, e.g., leader of a country, president of a firm, chief at the scene of a fire. Play of a game is usually divided into moves, each of which begins with the presentation of information that players are asked to accept as true and to use as a basis for their deliberations and decisions. This information is called the scenario for the game. In the terminology used above, the game would be preceded with a presentation of the context and the crisis up to the beginning of the game. The playing of the game usually involves another set of persons (or intelligent agents) who administer the game. They are commonly called controllers or referees and usually include those who designed the game and those who will analyze its results. Games have usually been played with all participants at one site; however, distributed games can be played with remotely located players communicating via computer networks, video conferencing, or other means, or across a whole set of federated environments.

Figure 2 shows a roadmap for gaming that covers progress to date from stand alone gaming through potentially

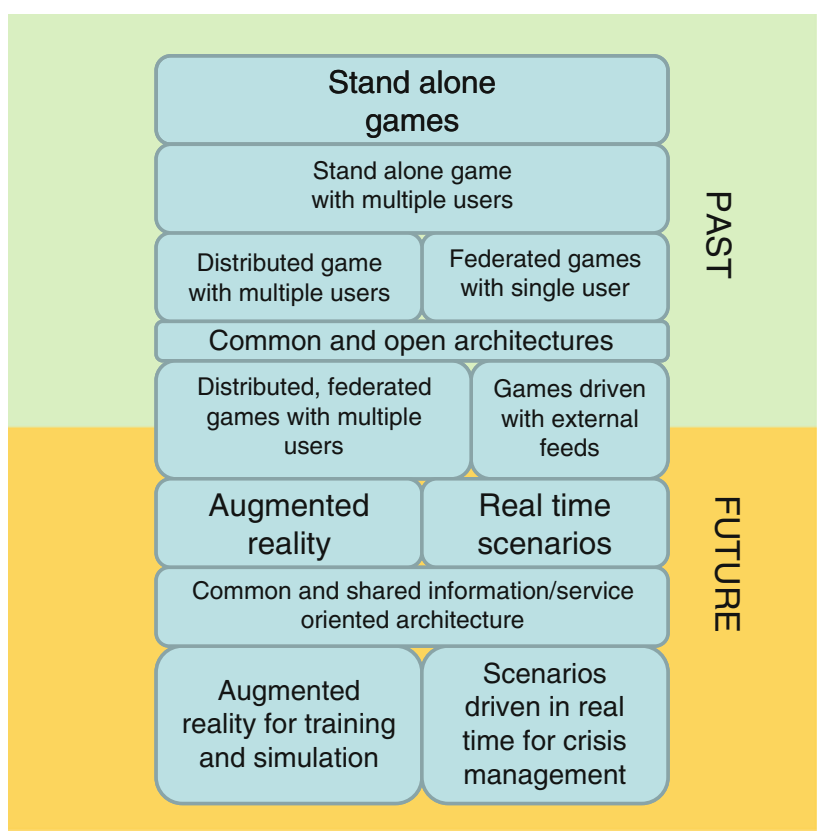

Fig. 2 Gaming and crisis management roadmap

widely distributed, real-time environments for gaming in the future. Terms within this roadmap are further discussed in later sections.

\subsection{Why use gaming?}

Gaming can be used for many purposes. Shubik (1971) divides these into six categories. Crisis management games can be designed to achieve four of these:

- Teaching. One of the major uses of gaming has been as a motivational aid to learning. Business games are used extensively in business schools for this purpose. Gaming has been found to attract the players' attention and involve them deeply. It is an extremely useful way to learn and organize facts. And, because of a game's logical consistency and completeness, it is a useful device for encouraging students to think in terms of models and abstractions. It is also a useful device for teaching about interpersonal relations, such as the need for cooperation, communication, negotiation, and compromise.

- Training. Games can be used to improve the performance of a group of persons in an organization in carrying out their normal jobs ("off line", so that mistakes do not affect actual outcomes, or as we discuss below, increasingly in real time to enable better decision support). More important for crisis management, however, is that games can be used as "dress rehearsals", just as in the theater. In this case, they are aimed at preparing for coordination of the players who 
may have to cooperate in team action on a temporary basis.

- Operations. Operational games are used by military, governmental, and corporate organizations for contingency planning, strategy exploration, and system testing. In this type of use, the game can reveal errors or omissions in a strategy, explore assumptions and uncover those that are implicit, examine the feasibility of an operational concept, identify areas in which required information is lacking, and suggest areas needing further attention.

- Experimentation. Human beings are more difficult to experiment with than rats or guinea pigs. In experimental gaming, human decision-making behavior is studied by observing the performance of individuals in an experimental setting. Crisis games, for example, might be used to study decision making under stressful, overloaded, conditions.

Schelling [in (Levine et al. 1991)] is more specific about what he thinks crisis games can accomplish. Because of the relevance of his insights, we quote him at length:

Games are...awfully good at... demanding careful sequential analysis of plans, decisions, events, and intelligence. Very few plans or situations seem to be subjected to a process of 'walking through', of dress rehearsal. This is particularly true of plans and contingencies that are political-military, i.e., that involve...communications, intelligence activities and interpretation, and the coordination of activities over time as well as among agencies. Crisis games typically subject the players to a continuous process over time in which they are both making decisions and living with prior decisions, in only partial control of their environment, committing themselves to actions that have lead times, reaching decisions on the basis of intelligence that is only partially available when they cannot wait for more. People sensitive to a variety of responsibilities collaborate, applying the criteria that are relevant to their own interests, making estimates that reflect their own kinds of knowledge, and putting themselves in a mood to worry about probabilities rather than just a list of possibilities. They really live through a simulated crisis and not only learn things about their plans and their predictions but learn something about the nature of crisis.

\section{The use of scenarios and gaming as methodological tools to improve crisis management}

Advances in computers, networking, and telecommunications have opened up new possibilities for using gaming as a methodological tool for improving crisis management. It is becoming easier to develop models to support games, to have players at distributed workstations interacting with each other, to have automated controllers supply exogenous events to the players, to enable players to query online data files during the game, and to prepare presentation graphics for use during the game and for post-game debriefings. Multimedia techniques can be used to present scenario updates to players in "newscast" format and to present pre-recorded briefings by experts to players.

A number of crisis management (CM) game simulations have emerged recently that mix the elements discussed above in order to present an immersive training environment. Examples of two such systems include the following:

1. Advanced Disaster Management Simulator (ADMS) by ETC. Simulation-A virtual reality system used to immerse an incident commander into a simulated crisis management situation. A combination of an immersive $3 \mathrm{D}$ environment and CM simulation that places the incident commander (and various role players) within the scene.

2. Incident Commander ${ }^{\mathrm{TM}}$ by Breakaway Ltd-A 2D, map based, top down CM game that puts the player in the role of an incident commander handling a wide variety of CM scenarios. The player has to co-ordinate the numerous agencies to respond to the emerging crisis.

Many organizations with responsibility for crisis management are adopting these new technologies for use in constructing a crisis management system (CMS) to coordinate response to a crisis, provide decision support during a crisis, and support activities prior to the crisis and after the crisis. If designed correctly, that same CMS could be easily used in a simulation mode to play a crisis management game. (Such a use of the system would also provide personnel with opportunities to rehearse for real crises using the same tools they would have available to them in a real crisis.) This is the approach recommended by Walker et al. (1989). In addition to providing the "look and feel" of the real-world events, such systems have an attractive cost-benefit ratio, since they can reduce classroom training costs and eliminate the liability costs in using people and equipment in live simulations.

Based on the generic purposes for gaming given in the previous subsection, we can see five specific purposes for using gaming as a tool for improving crisis management:

1. To assist in pre-crisis resource requirements determination and resource allocation decision making;

2. To assist in response planning;

3. To assist in training in crisis management for actual crises; 
4. To manage an ongoing crisis in real time;

5. To analyse and re-run a crisis after resolution to learn from experience and modify the CMS for future deployment.

Resource requirements and resource allocation depend crucially on the specific crisis situation, and as we are recommending, driving scenarios from real-time feeds and data flows can be used to rapidly assess developing requirements. As we suggested above, while scenarios and games are not meant to be predictive, and there is an extremely low probability of any given scenario coming to pass, new technologies can allow a set of scenarios to be rapidly developed from real-time data feeds and honed to provide a representation for decision support during a developing crisis situation. Gaming can thus be developed as a better tool for response planning and a much more effective tool for training. If used for these purposes, the focus can be on lessons learned that to use the words of Schelling (Levine et al. 1991) "are not so particular as to depend on the locale of the crisis, the scenario chosen to initiate the game, the individual participants, or even the character of the crisis".

Based on their experiences in crisis games, Levine et al. (1991) conclude that "the main beneficiaries of the game are the participants, and,... in the case of participants in decision-making positions, the benefits are likely to be high". Thus, the game players should ideally be the same persons who will be in training, and they should be using the same support tools (databases, communications facilities, computers) that they would be using during the real crisis. They would then discover facts, ideas, possibilities, capabilities, and arguments that would be valuable for the real crisis (e.g., resource constraints, data availability, jurisdictional problems, standard operating procedures, relevant telephone numbers). Of course, their actions might still not be the same as they would be if the crisis were real, since they will not be experiencing the actual tensions and pressures. But having the CMS and operating it in real time should come close to reproducing the real situation.

Weiner (1968) describes what is involved in preparing, playing, and analyzing a game. The preparation phase starts with the definition of the purpose of the game. The purpose drives the specification of scenario required, the players to include, the data to prepare, and the analyses that will be done afterward. Once the purpose is established, a scenario can be prepared, which must include the context (geography, location of resources, lines of communication, etc.) and the crisis (events before the start of the game and exogenous events during the game). Having developed the inputs, the next major part of preparation is to develop the rules of the game. These rules include political restrictions, operating procedures, etc., that are the ground rules given to the players. They also include rules that will be used in the CMS (which will play the role of the controller or referee for the game) for estimating the effects that the players' actions will have on the system's performance. The CMS will use these rules to create the dynamic changes in the crisis situation to which the players will have to react over time. Typically, these rules should be very close to the set of rules used for real crisis management, but a significant level of experimentation is possible to test new ways of responding to a real crisis.

Using the rules for the CMS, a simulation system must be generated that will provide the game control. If the gaming use of the CMS has been thought of before the system is built, there will be built-in mechanisms that will facilitate the programming of the game. In fact, many of the capabilities needed for the simulation will be required capabilities of the CMS. Figure 3 shows a typical architecture that might be developed to enable the CMS to function and could strongly parallel the CMS generated for managing real-time crises.

Once all of the preparatory work has been completed, it is possible to play the game. As mentioned above, the players should be playing the roles that they would play in the case of a real crisis. The playing conditions should

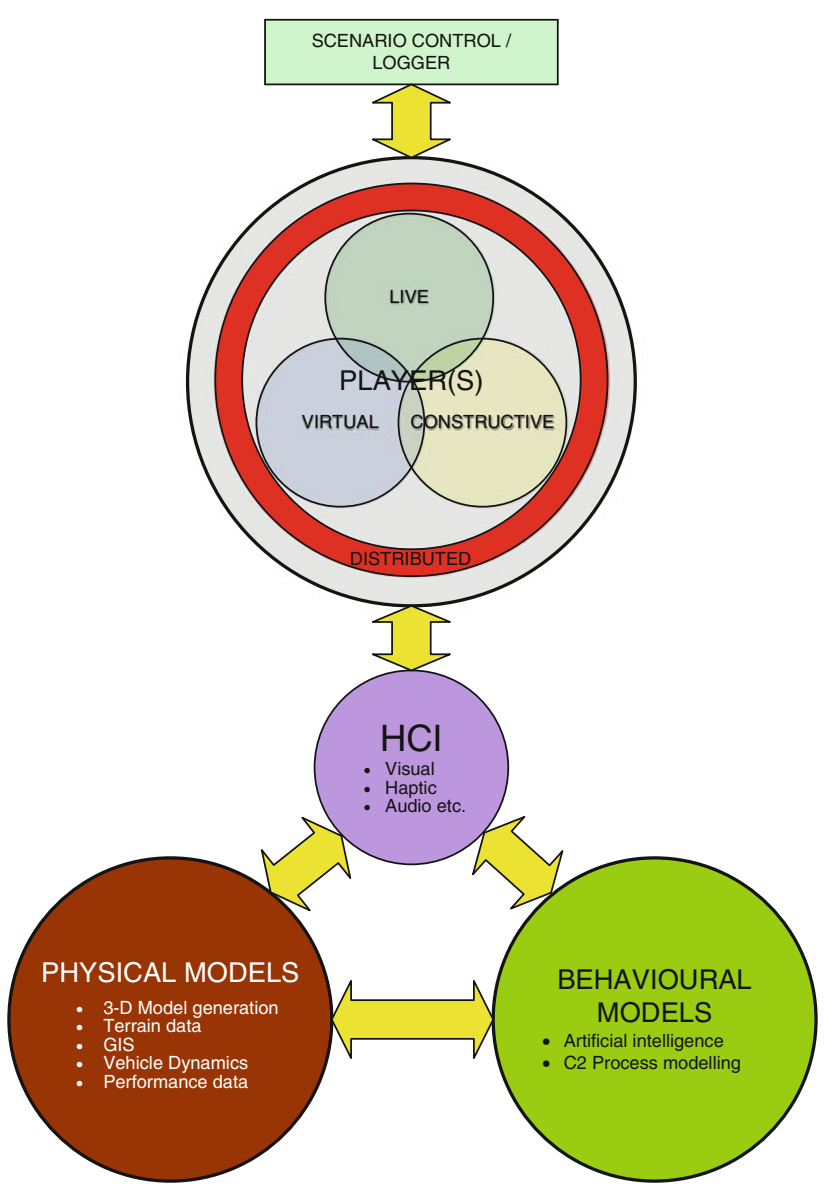

Fig. 3 Representation of a generic simulation/CMS environment 
match as closely as possible the conditions that would exist in a real crisis. Play is started by a "briefing" to the players (perhaps via multimedia feeds), which describes the context and the events or conditions that led to the crisis. Then, the precipitating event is announced and play begins. If serious confusion, errors, or arguments occur, the game can be stopped and restarted, but efforts should be made to finish the entire game without interruption. When play ends, the analysis phase begins. Since the type of analysis that will be done is determined by the objective or purpose for which the game is being played, it is not possible to describe specific analyses without describing specific games, although a common feature will be a capture of relevant information throughout the gaming - in an identical way in which data capture would take place during a real crisis. It is possible, however, to describe typical kinds of analyses.

One type of analysis is outcome oriented. It examines what took place and evaluates the performance. Positive and negative actions are noted, as well as actions that might have helped, but were not taken. The outcome-oriented analyses generally span the entire system and focus on overall effectiveness. There are also special analyses that focus on a single aspect of game play. For example, an analysis might be made of the actions of one particular agency (with the game replayed several times, to look at various alternative strategies for that agency). Another reason for replicating play might be to look at the effects of changing the resource allocation or information availability. An analysis might also be made of the behavior under stress of the various players.

Note that these analyses do not solve problems; if anything, they define new ones (e.g., holes in the system, missing information, inaccurate data in databases). It is also not necessarily true that if something happens in the game world, it will happen in the real world. As Levine [in (Levine et al. 1991)] warns, "the seductiveness of gaming is such that it is all too easy to turn hypotheses into conclusions". He then gives the following advice: "Game if you will. But in presenting policy results, don't tell anyone that you gamed. Present it in essay, model, or other analytical form without mentioning the game. If it is convincing in this form, then the game has been as good an instrument as any. If it is necessary to fall back on game "evidence", however, then the whole process is of very doubtful validity". One follow-up to a game might be specifying a field test to reduce uncertainty as to the validity of the game's results.

\section{An architecture for simulation and gaming}

The uses and objectives of crisis management games described above suggest the following architectural characteristics for the ICT system:
- An open architecture to enable existing and new elements of simulation and gaming to be federated and scalable;

- Standard and open architectures to allow real-world situations to be embodied in games and simulationsfor example, databases of light detection and ranging (LIDAR) scanned images, availability of physical descriptions of buildings and infrastructure [see, for example, (Mallet and Bretar 2009)], and representations of system interdependencies;

- A distributed architecture that may be facilitated over the Internet or over dedicated networks developed specifically for the gaming environment. Such environments are increasingly ubiquitous with the rise of online gaming;

- An architecture that allows the seamless introduction of "real-world" feeds, in order to enable augmented reality to be introduced to drive scenarios and enable real-world interaction.

Many of these features are included in the roadmap of Fig. 2.

A typical schematic architecture is shown in Fig. 4. As discussed above, we aspire to move toward a CMS architecture that is common to both the gaming and simulation environment, as well as to real crisis management and decision support. Utilizing cutting edge techniques, such as augmented reality, would enhance the look and feel of the simulated environment for training purposes and would enable the presentation of decision support information in novel forms in a real-world crisis management environment. Clearly, the ability to capture and deliver data in significant volumes and complexity would also enhance the realism.

The definition of a CMS architecture that is open, scalable, and flexible should allow the incorporation of real-world data (such as meta-tagged video footage) and simulated data (e.g., plume cloud modeling) within a training or decision support tool. This system architecture would be used to drive both "offline" simulations or games, as well as the decision support tools for real crises. This will also enable data from real crises to be captured for use in the analysis phase (after resolution and restoration) to better prepare the system for response during future events (see Fig. 1). We, therefore, suggest that an approach similar to the US Network Centric Warfare or UK Networked Enabled Capability be utilized to form the common and shared information picture.

Figure 5 is a schematic diagram for potential information management, exploitation, and assurance for our proposed simulation and gaming environment. The primary feature of this environment is the ongoing collation of data feeds prior to a simulation or a real-time crisis management event, in order to develop a shared situational knowledge 
Fig. 4 Potential gaming architecture

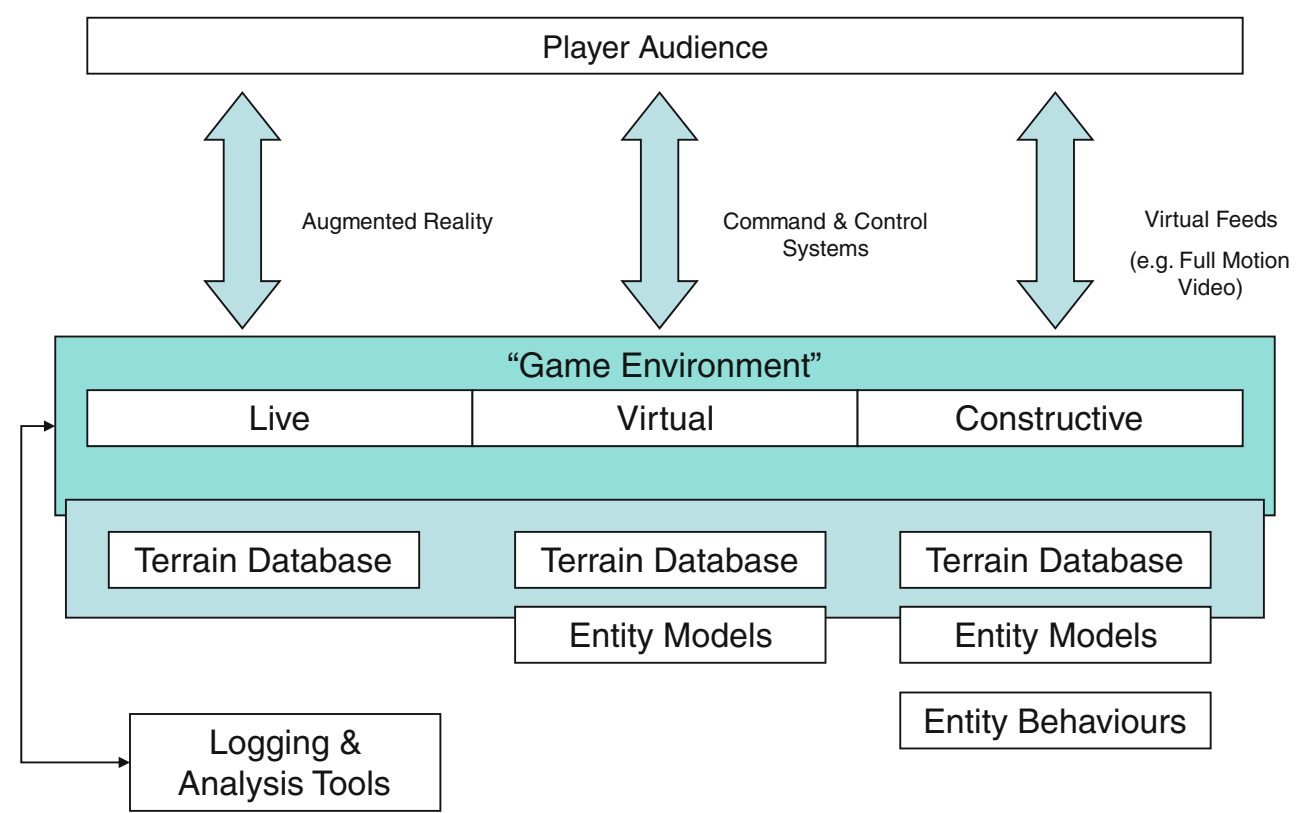

base or common and shared information picture for either. This accords with the left-hand side of Fig. 1. During the transition to either a simulation or a real-time crisis, the same knowledge base transitions (scenario independent to scenario dependent) will then be performed for information exploitation to manage the ongoing environment. In this scheme, information is continually captured to feed the knowledge base, which can then be exploited for learning from experience, re-running simulations or real-time crises, and identifying required capability or capability gaps.

\section{Benefits and barriers to realizing gaming and simulation environments}

To summarize, we see two major benefits from simulation and gaming in realistic environments. They:

1. Allow experimentation in nearly real-life situations in laboratory simulations when it would be more costly, difficult, or impossible to create such experiments in the field;

2. Support excellent visualizations that help explain the simulations to decision makers and to involve a range of people in analyses, learning, and decision making.

The features that produce these benefits are that they:

- Enable people, intelligent agents, and real (live) equipment to interact with realistic models, simulations, and visualizations;

- Allow a range of different players to share a consistent representation of the issues being looked at and to work concurrently to develop and assess solutions;
- Permit effective visualization and manipulation (the ability to answer "what if" questions) of problem situations and alternative possible solutions;

- Are flexible - both the simulation and the players may be represented at different degrees of scope and detail. Components may be modified relatively easily to permit experimentation;

- May be run in real time or faster/slower as required;

- May be paused or re-run more easily than is the case with an equivalent activity in the real world, permitting experimentation (free-play), iteration, sensitivity analysis, and on-the-fly adaptation;

- Allow the economical replication and re-use of components.

At the same time, there are barriers to delivering the proposed step change in the architecture and environment for simulations and gaming. These include:

- Participation-a simulation can require significant human participation to ensure the successful running of an event. Often the human participants need to be subject matter experts, who are not always available.

- Data - the fidelity of a simulation/gaming environment is largely dependent on the quality of input data; in particular, the representation of the physical model might require a high-fidelity terrain database. Access to the data, or transferring it to the correct format, can often form a significant barrier.

- Validation-due to the number of complex interlinked parts that go to form a simulation environment, it can be difficult to fully validate the results of a gaming activity. In particular, when human decision making 


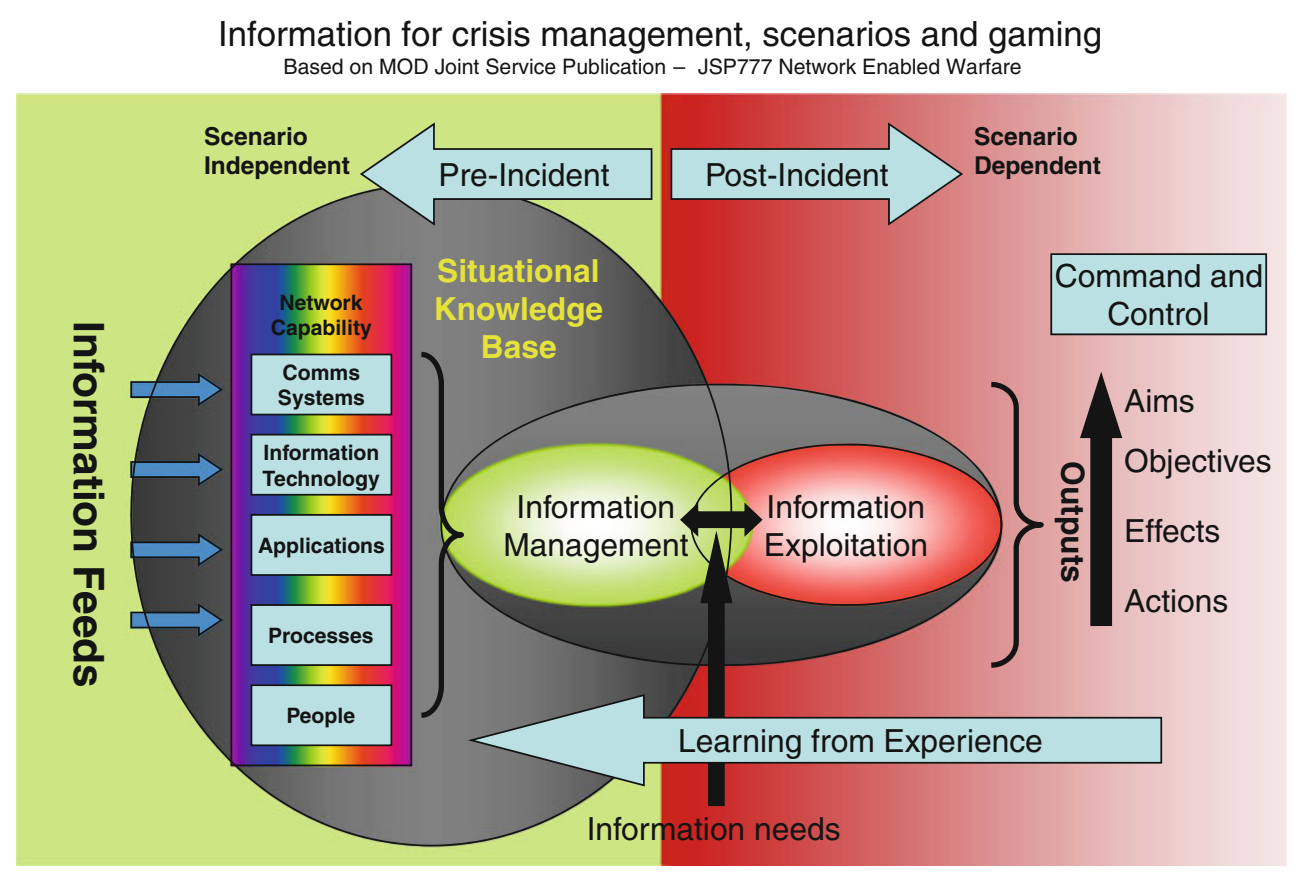

Fig. 5 Information management and exploitation architecture, based on MOD JSP777, Network Enabled Warfare

forms part of the simulation activity, it can be difficult to use traditional validation and verification techniques.

- Behavioral representation-the performance of any simulation is mostly dependent on the component sub-models from which it is constructed. If elements of the chain are weak, the model will also be weak. It is important that the basis of representation should be understood and well explained.

- Computing power-depending on the structure of a simulation, considerable computer resources can be required. However, this is becoming less of a barrier as computers become more powerful and as software efficiency improves, partly through integration of novel elements from computer game and web developers.

- Scepticism-some people will not take simulations seriously, thinking they are little more than games. Others question how close they can be to reality (see "behavioral representation" and "validation", above).

- Culture representation-in a simulation that aims to represent a complex environment such as an urban scene, it is important to represent realistically the cultural elements of that environment, such as pattern of life activities within a city, commuting traffic, crowd behavior. This cultural representation, though possible, can be difficult to achieve, mainly due to it being computationally intensive. In addition, capturing accurate information about culture, including the rapidly changing social mix, can require considerable, and sometimes difficult, research.
The authors are convinced that the boundary between gaming and reality will become ever closer. With sensors becoming ubiquitous in the environment, networked via the Internet and mobile infrastructure, the availability of data feeds to drive a real-time gaming environment is becoming more and more standard. The authors anticipate that the rise in nomadic computing devices (such as the Android and iPhone) will drive this trend and raise the potential for simulations and games to be led by real-time environments that enable the game players or actors to experiment with decision making in a benign but realistic real-world environment. During actual crisis management operations, the transition then becomes seamless for those required to make real-time decisions in a real-world environment that uses familiar interfaces to the data and decision-making space.

\section{Conclusions}

The above discussion suggests that simulation and gaming can play a useful and even pivotal role in crisis management planning and training and its real-time delivery. In particular, ongoing developments in information technology and telecommunications afford an opportunity to use these methodological tools in ways that were not previously possible. In the past, games were extremely expensive to stage and took a great deal of time to play. Because much of the work had to be performed manually, very few 
"moves" could be carried out. If an existing CMS were able to be used offline to support the game, many of the game's support requirements would already exist, and most of the work that had to be carried out manually could be automated. Operating in real time but offline, crisis managers would be able to use the computer systems, databases, and communication channels that they would have to use in an actual crisis situation. The marginal costs are likely to be relatively small and the potential benefits large. The time is ripe to try such a creative, state-of-the-art approach.

Open Access This article is distributed under the terms of the Creative Commons Attribution Noncommercial License which permits any noncommercial use, distribution, and reproduction in any medium, provided the original author(s) and source are credited.

\section{References}

Abt CC (1970) Serious games. The Viking Press, New York

Beroggi GEG, Waisel L, Wallace WA (1995) Employing virtual reality to support decision making in emergency management. Saf Sci 20:79-88

deLeon P (1975) Scenario designs. An overview. Simul Games 6(1):39-60

DeWeerd HA (1967) Political-military scenarios. The RAND Corporation, Santa Monica, p 3535

DeWeerd HA (1973) A contextual approach to scenario construction. The RAND Corporation, Santa Monica, p 5084

Geisler MA, Ginsberg AS (1965) Man-machine simulation experience. The RAND Corporation, Santa Monica, p 3214

Helmer Olaf (1966) Social technology. Basic Books, New York

Kahn H, Wiener A (1967) The year 2000-a framework for speculation on the next 33 years. Macmillan, New York
Kleiboer M (1997) Simulation methodology for crisis management support. J Conting Crisis Manag 5(4):198-206

Levine R, Schelling T, Jones W (1991) Crisis games 27 years later. The RAND Corporation, Santa Monica, p 7719

Mallet C, Bretar F (2009) Full-waveform topographic lidar: state-ofthe-art. ISPRS J Photogramm Remote Sens 64:1-16

Mendonça D, Beroggi GEG, van Gent D, Wallace WA (2006) Designing gaming simulations for the assessment of group decision support systems in emergency response. Saf Sci 44: $523-535$

Quade ES (1985) Predicting the consequences: models and modeling, Chap. 7. In: Miser HJ, Quade ES (eds) Handbook of systems analysis: overview of uses, procedures, applications, and practice. Elsevier Science Publishing Co., Inc., New York

Quade ES (1989) Analysis for public decisions, 3rd edn. Elsevier Science Publishers, Amsterdam

Schwabe W (1994) An introduction to analytic gaming. The RAND Corporation, Santa Monica, p 7864

Schwarz B (1988) Forecasting and scenarios, Chap. 9. In: Miser HJ, Quade ES (eds) Handbook of systems analysis: craft issues and procedural choices. Elsevier Science Publishing Co., Inc, New York

Shubik M (1971) On the scope of gaming. The RAND Corporation, Santa Monica, p 4608

Shubik M, Brewer GD (1972) Models, simulations, and games-a survey, R-1060- ARPA/RC. The RAND Corporation, Santa Monica

Tate DL, Sibert L, King T (1997) Using virtual environments to train firefighters. IEEE Comput Graph Appl 17(6):23-29

Walker JA, Ruberg GE, O’Dell JJ (1989) Simulation for emergency management: taking advantage of automation in emergency preparedness. Simulation 53(3):95-100

Weiner MG (1964) Gaming methods and applications, Chap. 11. In: Quade ES (ed) Analysis for military decisions. North-Holland Publishing Co., Amsterdam

Weiner MG (1968) Gaming, Chap. 14. In: Quade ES, Boucher WI (eds) Systems analysis and policy planning: applications in defense. Elsevier Publishing Co, Amsterdam 